\title{
RECOMBINATION RADIATION IN THE CESIUM POSITIVE COLUMN
}

\author{
By F. L. Mohler
}

ABSTRACT

The radiation emitted along the axis of a positive column has been measured by spectrophotometric methods and electrical measurements have been made with small probe surfaces near the line of sight.

The continuous spectrum near the $6^{2} \mathrm{P}$ limit was measured with pressures ranging from $0.8 \mu$ to $290 \mu$ and with a 50 -fold range of electron concentration, $N_{e}$. At low pressures the intensity varies as $N^{2}$ e and is nearly a linear function of pressure with about twice the zero pressure value at $9 \mu$ for $N_{e}=10^{12}$ or less. At higher pressures and high values of $N_{e}$ the increase in intensity is less than $N_{e}^{2}$.

The intensity near the $5^{2} \mathrm{D}$ limit remains nearly equal to that at $6^{2} \mathrm{P}$ for pressures above $3 \mu$. At high pressures the subordinate and fundamental series lines change in the same way as the continuous spectrum.

The low-pressure measurements give a basis for evaluating the probability of spontaneous recombination of an ion and an electron into the $62 \mathrm{P}$ level. The effective collision area for 0.3 volt electrons is $1.7 \times 10^{-21} \mathrm{~cm}^{2}$.

The effect of vapor density is to increase the probability of transition. Expressed as a 3-body collision, recombination takes place if an electron and an atom are simultaneously within $6 \times 10^{-8} \mathrm{~cm}$ of an ion.

With high electron concentration radiation is suppressed in a process which is probably the converse of ionization by collision. This quenching process takes place when two electrons are simultaneously within a distance of about $10^{-7} \mathrm{~cm}$ of an ion but the distance increases with vapor density.

\section{CONTENTS}

I. Introduction

II. Experimental procedure

III. Results

1. Intensity of the $6 \mathrm{P}$ band

2. Intensity of other bands and lines....

IV. Discussion

1. Spontaneous recombination

2. Vapor density effect.

3. Quenching of recombination by electron collisions

4. Conclusions

\section{INTRODUCTION}

This is one of a series of papers giving measurements of the atomic constants which determine the rate of interaction of electrons, ions, and atoms in an electric discharge. ${ }^{1}$ The uniform glow which fills most of the tube in the familiar type of electric discharge is a mixture of atoms, positive ions, and negative electrons. The electrons collide with the atoms and ions and produce ionization and radiation. The

1 Mohler, B.S. Jour. Research, vol. 9 (RP455), p. 25, 1932: (R P485), p. 493, 1932.

Boeckner and Mohler, B.S. Jour. Research, vol. 10 (RP 535), p. 357, 1933. 
chance of producing any given effect can be expressed in terms of an area around each atom through which an electron must pass to produce the effect. These collision areas are evaluated by measuring the number of atoms or ions, the number of electrons and the total effect produced in a given volume. This paper deals with collisions in which an electron becomes attached to an ion to form a neutral atom and the effect measured is the continuous spectrum radiation emitted in this recombination process. Results show that this effect which is negligible in low-pressure discharges can become an important factor in high-pressure high-current discharges.

When a positive ion and an electron recombine spontaneously, that is, in a 2-body collision, the energy will be radiated in a single quantum of energy

$$
h \nu=h \nu_{i}+1 / 2 m v^{2}
$$

where $\nu_{i}$ is the frequency of a series limit and $v$ is the electron velocity. With electrons of random velocity there is a continuous band extending beyond each series limit and the intensity at any frequency measures the rate of recombination of electrons of the corresponding speed into a particular level, provided the continuous bands do not overlap. The rate of recombination will be expressed as an effective area $q\left(\nu_{i} v\right)$ characteristic of the limit $\nu_{i}$ and the electron speed. The number of collisions within this area gives the number of quanta radiated

$$
\frac{J(\nu) d \nu}{h \nu}=q\left(\nu_{i} v\right) N^{+} N_{e} v f(v) d v
$$

where the $N$ 's are the number of ions and electrons per cubic centimeter and $f(v) d v$ is the fraction of electrons having a range of speed $d v$ corresponding to $d \nu$. The electrons in a plasma have a Maxwell distribution of velocity and $N^{+}$and $N_{e}$ are very nearly equal

$$
\frac{J(\nu) d \nu}{h \nu}=q\left(\nu_{i}, v\right) N_{e}^{2} \frac{4 \pi^{-1 / 2}}{\sqrt{2 m e}} \frac{V}{V^{3 / 2}} \exp .\left(-V / V_{0}\right) d \nu
$$

where $V$ and $V_{0}$ are the electron energy and the most probable energy in electron volts.

About five years ago Boeckner and the author made a quantitative study of the continuous bands emitted by a cesium discharge. ${ }^{2}$ Results indicated that the intensity distribution depended on the electron velocity distribution in a manner consistent with equation (2) and for the $6^{2} \mathrm{P}$ and $5^{2} \mathrm{D}$ bands $q$ was inversely proportional to $\nu^{2} V .^{3}$ The intensity distribution ${ }^{4}$ then has the form

$$
\lambda J(\lambda)=b N^{2}{ }_{e} \exp \cdot\left(-V / V_{0}\right) / V^{3 / 2}{ }_{0}
$$

where $b$ is a constant characterisitc of the limit. As concerns the absolute magnitude of the effect, the results indicated complications in the theory. The variation of intensity was less than $N^{2}{ }_{e}$ for high values of $N_{e}$ and there was a marked increase in intensity with vapor pressure. The vapor pressure does not enter explicitly in equation (2) and the only predicted effect would be a slow variation resulting from the small change in $V_{0}$. Nevertheless the importance of vapor pres-

\footnotetext{
Mohler and Boeckner, B.S.Jour. Research, vol 2 (R P46), p. 489, 1929.

${ }^{3}$ We here use total quantum numbers instead of the Paschen notation used in other papers of this series. $6 \mathrm{P}$ and $5 \mathrm{D}$ are the first $\mathrm{P}$ and $\mathrm{D}$ terms of cesium.

4 Boeckner, B.S.Jour. Reaserch, vol. 6 (RP274), p. 277, 1931.
} 
sure has been emphasized in numerous qualtitative studies of recombination spectra. ${ }^{5}$

Recently Webb and Sinclair ${ }^{6}$ have studied the afterglow of a mercury discharge as a function of electron concentration and electron temperature. The intensity was proportional to the first power of $N_{e}$ and decreased very rapidly with increasing electron temperature. The results could be accounted for by assuming that recombination proportional to $N^{2}$ e was quenched by collisions of electrons with energy exceeding about 1 volt. They also found a rapid increase in intensity with pressure.

\section{EXPERIMENTAL PROCEDURE}

Mohler and Boeckner used the negative glow and dark space between a hot wire cathode and surrounding anode as a source of radiation but the positive column is better adapted to the purpose. The radiation per unit volume is somewhat less but the volume can be made as large as desired and is not a function of the pressure. The procedure was to measure the intensity sighting along the axis of the column and to measure the electron concentration with small probe surfaces at the axis.

The discharge tube was $1.8 \mathrm{~cm}$ in diameter and the length of the column was about $24 \mathrm{~cm}$ along the line of sight. The thermionic cathode and anode were in side tubes. The cesium was in an appendix and the vapor pressure was controlled by a separate heater around it. The probe surfaces were the ends of platinum wires covered except at the end with glass. Commonly there were two probes 0.4 and $1 \mathrm{~mm}$ in diameter.

The technique of the electrical measurements has been described before ${ }^{7}$ but will be repeated in part as these measurements offer the chief source of uncertainty. The electron current reaches a nearly constant value at potentials positive to the space. The current per unit collecting area $I$ is then related to the electron concentration by the equation

$$
N_{e}=3.75 \times 10^{11} I / \sqrt{ } V_{0}
$$

and the electron energy $V_{0}$ is obtained in the usual manner from the slope of the linear $\log I$ vs. $V$ curve for values of $V$ negative to the space potential. The difficulty is that, while the random space current may be as high as 40 amperes per square centimeter, the probe current should not exceed 0.1 ampere which necessitates a small area. In cesium vapor it is necessary to avoid a close fit of the insulator around the probe to reduce leakage, and in use the probe is heated enough to cause a gradual change in the exposed area. 'The use of probes of two sizes reduces the uncertainty, as the effective area of the smaller one can be found by comparison. Absolute values are based on the mean results obtained with several tubes. An upper limit of pressure and current is set by the probe limitations; the lower limit is determined largely by intensity considerations.

Intensity was measured by direct comparison of the spectra of the discharge and of a calibrated tungsten strip lamp. The discharge was

\footnotetext{
8 Krefft, Zeits. f. Phys. ,vol. 77, p. 752, 1932.

- Webb and Sinclair, Phys. Rev., vol. 37 , p. 182, 1931

7 Mohler, F. L. Collisions of the First and Second Kind in the Positive Column of Cesium Discharge,

B.S.Jour. Research, vol. 9 (RP485), p. 493, October 1932.
} 
focussed on the strip and the superposed images of the strip and discharge were focussed on the spectrograph slit. Visual measurements were made by varying the strip lamp current to match the discharge. Photographic measurements were based on densitometer measurements with the strip and discharge nearly matched and were always more accurate than my visual measurements. A correction for transmission of the tube and furnace windows and first lens is included. The accurate collimation of points extending $24 \mathrm{~cm}$ along the line of sight would require a very small aperture and the actual aperture ratio of about one thirtieth is necessarily a compromise.

A troublesome source of error in the continuous spectrum measurements is the scattered light from the line spectrum. At 4,950 A the photographic fogging comes largely from the doublet at 4,555 and $4,593 \mathrm{~A}$. This is an absorption doublet and a bulb of dense cesium vapor in front of the discharge, combined with a mask over the plate, seemed to eliminate the trouble. Repeated runs were vitiated before the need of extreme precaution was realized.

\section{RESULTS}

\section{INTENSITY OF THE 6P BAND}

Figure 1 gives plots of the log of the intensity at 4,950 A, near the $6 \mathrm{P}$ limit, versus the log of the electron concentration at nine different vapor pressures. A correction of 0.23 must be added to give the intensity in the tube. Circles give a single set of measurements, judged to be the most reliable; but the interpolation and extrapolation are based on several other series of measurements. At low pressures the curves are straight lines of slope two, which means that the intensity is proportional to $N^{2}{ }_{e}$. Except at the highest pressures the curves start with a slope of two. At the lowest pressures the intensity is nearly independent of pressure while above $6 \mu$ the intensity is roughly proportional to the pressure at constant $N_{e}$.

Probably the intensity depends on the vapor density or number of atoms per cubic centimeter rather than on the pressure. A rough measurement with tube temperatures of $240^{\circ}$ and $440^{\circ} \mathrm{C}$., and a vapor pressure of $12.5 \mu$ showed that the intensity was about 35 percent less at the higher temperature. The density change would account for a 25 percent change and the discrepancy can be ascribed to inadequate control of the cesium temperature. An effect depending on collisions between atoms and relatively high-speed ions would vary with the density rather than the pressure.

The intensity distribution in the $6 \mathrm{P}$ band has been checked at two extreme pressures by intensity measurements at two wave lengths. From equation (3) the intensities at two wave lengths, $\lambda_{1}$ and $\lambda_{2}$, separated by an interval corresponding to $V_{2}-V_{1}$ electron volts, are related to the electron energy $V_{o}$ as follows

$$
\frac{\left(V_{2}-V_{1}\right) \log \epsilon}{\log \lambda_{1} J\left(\lambda_{1}\right)-\log \lambda_{2} J\left(\lambda_{2}\right)}=V_{o}
$$

$\Lambda$ t a pressure of $290 \mu$ with a 2 -ampere discharge, $V_{o}=0.187$ volts. Measurements of the reversal temperature of the resonance line give $V_{o}=0.195$ volts. At a pressure of $6 \mu$ measurements on a 3 -ampere discharge gave $V_{o}=0.255$ volts while electrical measurements and 


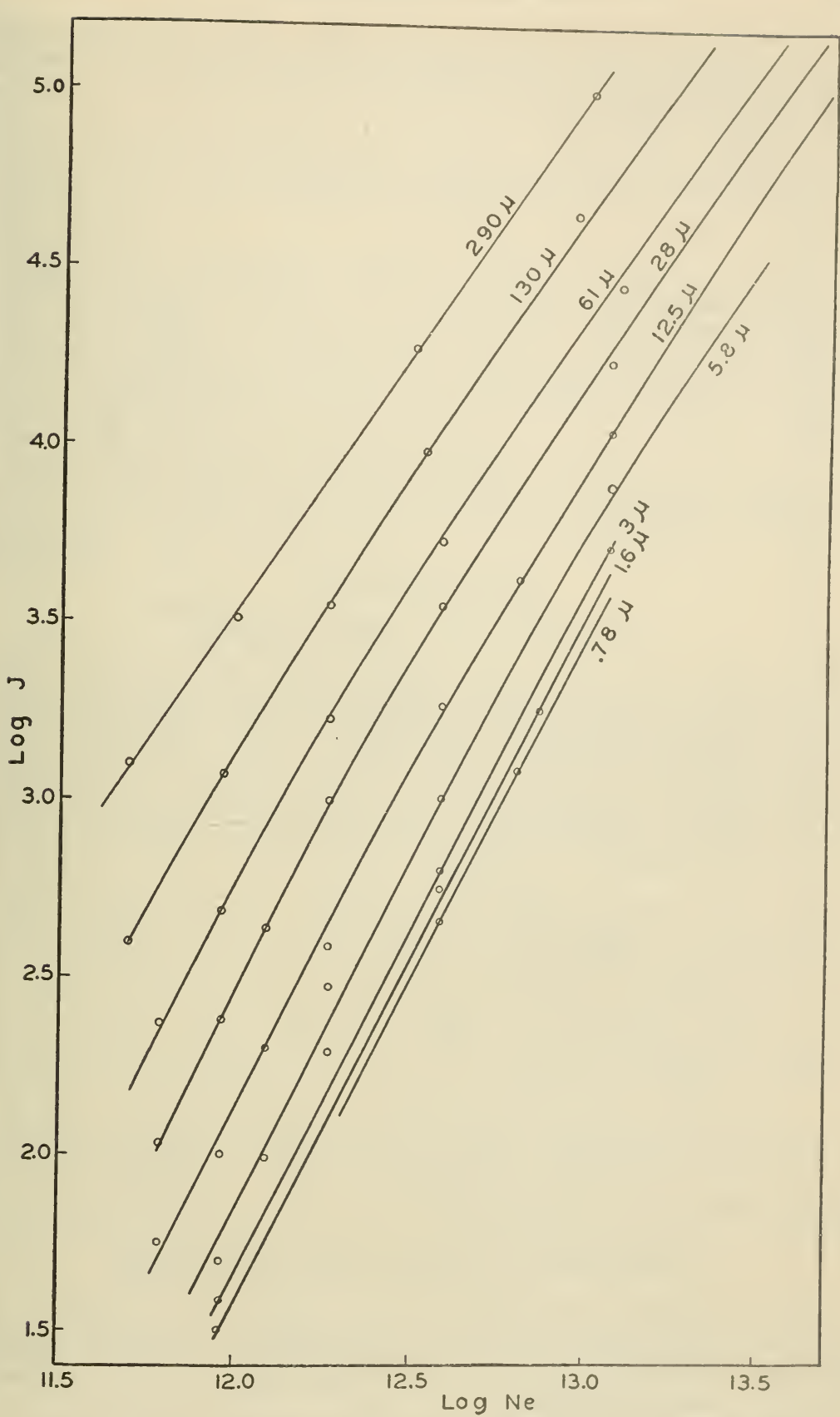

Figure 1.-Intensity of the continuous spectrum near the $6 P$ limit.

$\log J(4,950)$ versus the $\log$ of the electron concentration at nine pressures. $J$ is radiation flux in ergs in a wave length range of $1 \mu$ in an angle $2 \pi$. 
reversal temperature both gave $V_{o}=0.283$. The discrepancy may well come from window absorption and the conclusion is that the intensity distribution conforms closely to the predicted form over a wide range of conditions.

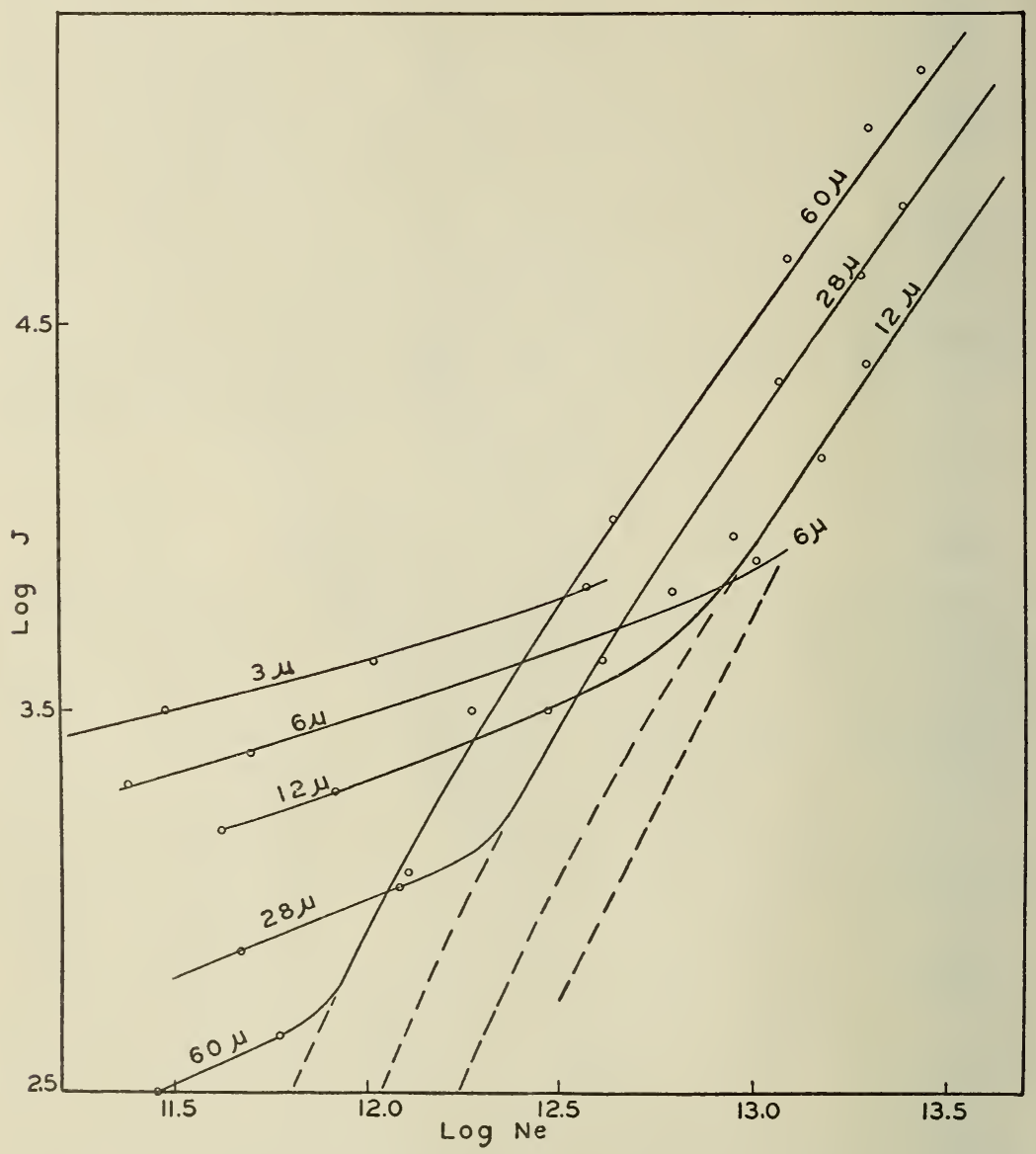

FIgURe 2.-Intensity of lines $6 P_{1 / 2}-10 D$ and $6 P_{3 / 2}-11 D$ and of continuous spectrum.

The steep, smooth curves give the continuous intensity, the dots and nearly horizontal curves the line intensity.

\section{INTENSITY OF OTHER BANDS AND LINES}

Visual measurements of the continuous spectrum at 5,925 A near the $5^{2} \mathrm{D}$ limit at pressures above $3 \mu$ show nearly the same variation with pressure and current as the $6 \mathrm{P}$ band and the absolute value is about the same as at $4,950 \mathrm{~A}$.

Visual settings were made on the superposed pair of lines $6 \mathrm{P}_{1 / 2}-10 \mathrm{D}$ at $5,466 \mathrm{~A}$ and $6 \mathrm{P}_{3 / 2}-10 \mathrm{D}$ at $5,503 \mathrm{~A}$. At pressures above $80 \mu$ the variation of intensity with pressure and current is the same as for the continuous spectrum. Figure 2 shows the results at lower pressures. The smooth steep curves give the intensity of the $6 \mathrm{P}$ band; the dots 
and full lines give the line intensity reduced by an arbitrary factor to the same scale. At the lowest pressures there is no correspondence between the variation of line intensity and continuous intensity. At intermediate pressures there is an abrupt transition with increasing $N_{e}$ to a variation paralleling the continuous spectrum. The variation of line intensity with pressure at constant $\Lambda_{c}^{\tau}$ has a sharp minimum near the point where the line intensity curve meets the continuous spectrum curve.

Qualitative observations indicate that all subordinate series lines change in a similar manner, the transition current and pressure being somewhat higher for the lower series lines. Unpublished measurements of the spectrum intensity distribution in the negative glow show that above the transition range the intensity distribution in the whole visible spectrum except the principal series doublet remains unchanged as the pressure and current are changed.

Because there is a rapid change in relative intensity of continua and lines below the transition pressure there is a misleading visual impression that the continuous spectrum suddenly appears in this pressure range.

\section{DISCUSSION}

\section{SPONTANEOUS RECOMBINATION}

The results bear out the evidence of previous work that the intensity of the continuous spectrum depends on factors not included in the elementary theory. The effect of vapor density shows that recombination under discharge conditions is not purely a spontaneous recombination in a 2-body collision. Nevertheless the pressure effect becomes extremely small at the lowest pressures and there is a basis for evaluating the probability of spontaneous recombination at zero pressure.

The experiments are complicated by the fact that the electron energy depends on the vapor pressure and current. The variation with current is of the order of 10 percent and will be neglected. $V_{0}$ decreases with increasing pressure from 0.38 to 0.195 volts over the extreme range. As $V_{0}$ enters equation (2) as $1 / V_{0}^{3 / 2}$, values of the intensity will be multiplied by $\left(V_{0} / 0.3\right)^{3 / 2}$ to reduce them to a standard value of $V_{0}=0.3$ volts.

Figure 3 includes values of $J$ as a function of pressure up to $61 \mu$ for $N_{e}=10^{12}$ and values of $J / 100$ for $N_{e}=10^{13}$. The values for $N_{e}=10^{12}$ are a linear function of the pressure within experimental uncertainty. The two curves have nearly the same intercept at $p=0$ and the separation at higher pressures measures the departure from the $N_{e}{ }^{2}$ law of intensity variation. The mean value of the two intercepts gives $J=43$ in units of figure 1 for zero pressure.

It is convenient to evaluate the effective cross section for recombination $q(6 \mathrm{P})$ at an interval corresponding to 0.3 volts from the limit, viz, 4,400 $\mathrm{A}$, and, from equation $(3), J(4,400)=0.415 J(4,950)$. It is estimated that 0.28 of this comes from the $5 \mathrm{D}$ band. On this basis the number of quanta emitted in a cubic centimeter in unit frequency range is $0.66 \times 10^{-5}$ times the plotted values. The effective collision area $q(6 \mathrm{P}, 0.3$ volts $)$, is by equation (2), $1.7 \times 10^{-21} \mathrm{~cm}^{2}$. As cesium $6 \mathrm{P}$ has an effectivc quantum number of 2.35 it should be compared with a hydrogenic 2 quantum level. The theoretical value 
for recombination of 0.3 volt electrons into the hydrogen $2_{2}$ level is $3.66 \times 10^{-21} \mathrm{~cm}^{2}{ }^{8}$

\section{VAPOR DENSITY EFFECT}

The increase in the rate of recombination with vapor density can be expressed as an increase in $q$ with the number of atoms per cubic centimeter $N_{a}$. The upper curve of figure 3 gives the relation

$$
q-q_{0}=0.71 \times 10^{-14} N_{a} q_{0}
$$

where $q_{0}$ is the value at zero pressure, $1.7 \times 10^{-21} \mathrm{~cm}^{2}$. The transition probability is doubled at a pressure of $9 \mu$ or when the average distance

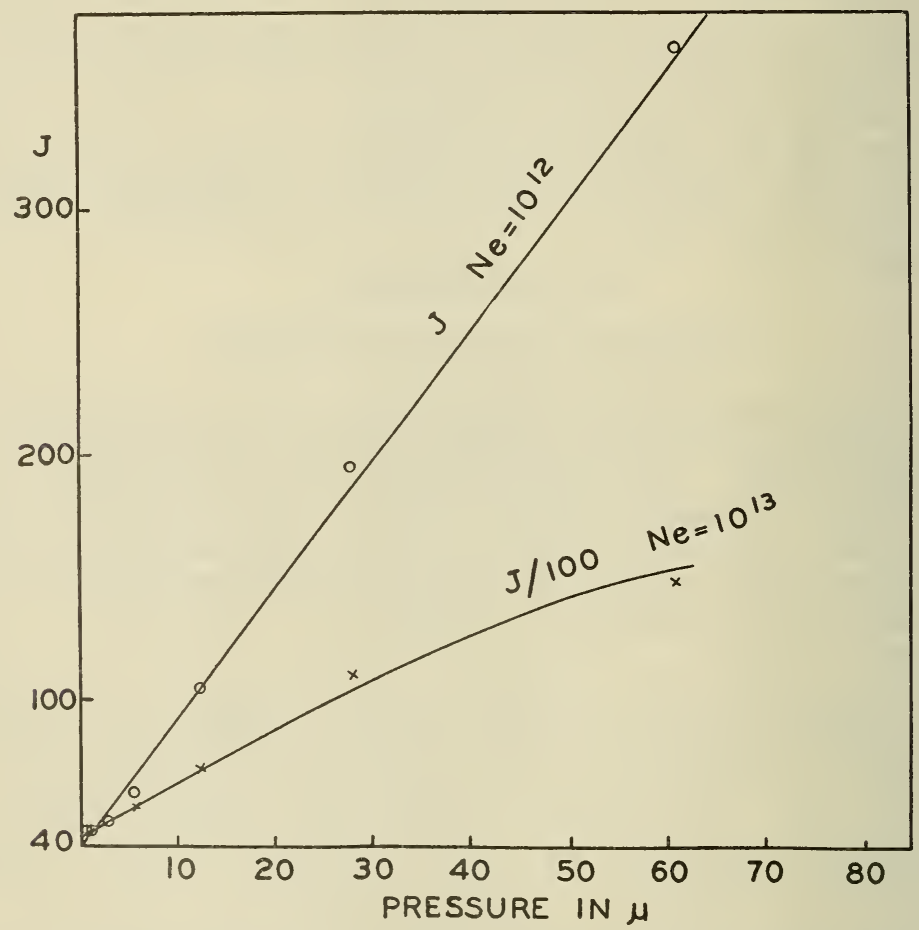

FIGURE 3.-Intensity of continuous spectrum at 4,950 A versus pressure. Intensity is reduced to $V_{0}=0.3$ volts.

between atoms is about $2 \times 10^{-5} \mathrm{~cm}$. The interaction can be described as follows: For an electron and ion to recombine spontaneously the electron path must have a narrowly restricted value of angular momentum relative to the ion. With the presence of another atom near the ion there is the possibility of the transfer of some of the momentum to that atom and the range of electron paths which can recombine is extended. The magnitude of the interaction is, however, surprisingly great.

$\Lambda$ s the interaction involves three bodies, one can express the probability of induced radiation as the probability of an atom and an electron being simultaneously within a distance $r_{a}$ of an ion. With

${ }^{8}$ Steuckelberg and Morse, Phys.Rev., vol. 36, p. 16, 1932. 
$N_{a}$ atoms per cubic centimeter the number of atoms within a distance $r_{a}$ of $N^{+}$ions is $\frac{4}{3} \pi r_{a}{ }^{3} N^{+} N_{a}$ and the number of electron collisions with these ions is $\frac{4}{3} \pi^{2} r^{5} N^{+} N_{e} N_{a} v_{e}$. At a pressure of $9 \mu, N_{a}=1.4 \times 10^{14}$, the number of induced collisions equals the number of spontaneous collisions.

$$
\begin{aligned}
& \frac{4}{3} \pi^{2} r_{a}{ }^{5} N^{+} N_{e} N_{a} v_{e}=1.7 \times 10^{-21} N^{+} N_{e} v_{e} \\
& r_{a}{ }^{5}=0.92 \times 10^{-36} \quad r_{a}=6.2 \times 10^{-8} \mathrm{~cm}
\end{aligned}
$$

This interaction distance $r_{a}$ bears no simple relation to the $q$ of equation (4), for equations (4) and (5) express two different interpretations of the phenomenon.

\section{QUENCHING OF RECOMBINATION BY ELECTRON COLLISIONS}

The continuous spectrum intensity increases less rapidly than the square of the electron concentration at high pressure and currents. This can be ascribed to an interaction between the recombining electron and a second electron in which the energy of recombination is given to the second electron. This process is the converse of ionization by electron collision except for the complicating effect of vapor density considered above. This quenching process is a 3-body collision and the number of collisions per cubic centimeter per second can be expressed in terms of the probability of two electrons being within a distance $r_{e}$ of an ion. The number of quenching collisions is proportional to $J_{0}-J$, where $J$ is the actual intensity and $J_{0}$ is the value that would be observed if $J$ were proportional to $N_{e}^{2}$. The absolute value can be expressed in terms of $q(6 P)$ of equation (4).

$$
\begin{gathered}
\frac{4}{3} \pi^{2} r_{e}^{5} N^{+} N_{e}^{2} v_{e}=\frac{J_{0}-J}{J_{0}} q(6 P) N_{e}^{2} v_{e} \\
\frac{4}{3} \pi^{2} r_{e}^{5} N_{e}=\frac{J_{0}-J}{J_{0}} q(6 P)
\end{gathered}
$$

Table 1 gives values computed for all curves where the departure from the $N_{e}^{2}$ relation was measurable. The interaction radius is of

\begin{tabular}{|c|c|c|c|c|c|}
\hline $\begin{array}{c}\text { Pressure } \\
(\text { in } \mu)\end{array}$ & $q$ (6P 0.3 & $\frac{J_{o}-J}{J_{0}}$ at $N_{0}=10^{13}$ & $\frac{4}{3} \pi^{2} r^{5} \cdot n$ 。 & $r_{\bullet}$ & 9.5 \\
\hline $\begin{array}{c}289 \\
130 \\
61 \\
28 \\
12.5 \\
5.8 \\
0\end{array}$ & $\begin{array}{c}\times 10^{-21} \\
107 \\
38 \\
14 \\
8 \\
4.2 \\
2.5 \\
1.73\end{array}$ & $\begin{array}{l}0.77 \\
.71 \\
.58 \\
.44 \\
.31 \\
(.18, \text { fig. 3) }\end{array}$ & $\begin{array}{c}\times 10^{-11} \\
23 \\
7.2 \\
2.2 \\
.96 \\
.36 \\
.125\end{array}$ & $\begin{array}{c}\times 10^{-7} \\
2.3 \\
1.8 \\
1.4 \\
1.2 \\
1 \\
.77\end{array}$ & $\begin{array}{c}\times 10^{-21} \\
2.1 \\
1.9 \\
1.6 \\
1.2 \\
.85 \\
.5\end{array}$ \\
\hline
\end{tabular}
the same magnitude as was found by equation (5) for radiation induced by atomic collision.

TABLE 1.-Quenching of recombination by electron collisions 
One can alternatively express the quenching in terms of the chance of a quenching collision during the recombination process by assuming a time interval $\tau$ and an effective area $q_{e}$ in which the collision of a second electron will be effective. There are $q_{e} N_{e} v_{e}$ collisions per second with a recombining atom and $q_{e} N_{e} v_{e} \tau$ is the chance of a collision given by $\frac{J_{0}-J}{J_{0}}$. From equation (6)

$$
q(6 P) q_{e} \tau=\frac{4}{3} \pi^{2} r_{e}^{5} / v_{e}
$$

The effective area $q_{e}$ can be expressed in terms of an effective radius $r^{\prime}$ and $\tau$ is naturally identified with the time it takes for an electron to cross this area. In conformity with the right side of equation (8) we can take $\tau=\frac{4}{3} r^{\prime} / v_{e}$. The radius $r^{\prime}$ is much larger than $r_{e}$ (at a pressure of $61 \mu$ it is $2.4 \mu 10^{-5} \mathrm{~cm}$ ). The difference is the same as appeared in the two views of the pressure effect. The right side of equation (8) expresses the chance of two electrons being within equal distances $r_{e}$ of the ion. The left side gives one electron the very much smaller distance corresponding to $q(6 \mathrm{P})$ so that $q_{e}$ must be proportionately larger. It is the product that is measured and there is nothing in the experiment to distinguish the two viewpoints. The values listed in the table are subject to a large experimental uncertainty for the effect is small except at pressures where the measurements of $N_{e}$ become inaccurate.

\section{CONCLUSIONS}

Recombination in a cesium discharge is in general not a spontaneous 2-body collision but is influenced by the presence of atoms and other electrons. From measurements at sufficiently low pressures one can evaluate the probability of spontaneous recombination. For 0.3 volt electrons recombining into $2^{2} \mathrm{P}$ the collision area is $1.7 \times 10^{-21}$ $\mathrm{cm}^{2}$ The effect of pressure is to increase greatly the probability of radiation. The effect of electron concentration is to quench the radiation. The interaction radii for the induced radiation resulting from atomic collisions and for the quenching by electron collisions are both of the same magnitude, $10^{-7} \mathrm{~cm}$, when expressed in terms of finding three bodies in a volume of radius $r$.

I am indebted to Dr. C. Boeckner for helpful suggestions concerning the interpretation of these results.

Washington, March 29, 1933. 